\title{
Toward Developing a Syllabus-Oriented Computer-Based Question-Banks Software to Support Partially Computerized Exams
}

\author{
Sulieman Bani-Ahmad \\ Department of Computer Information Systems, Faculty of Information Technology, Al-Balqa Applied University, \\ Salt, Jordan \\ Email: Sulieman@bau.edu.jo
}

Received 1 April 2015; accepted 19 May 2015; published 22 May 2015

Copyright @ 2015 by author and Scientific Research Publishing Inc. This work is licensed under the Creative Commons Attribution International License (CC BY). http://creativecommons.org/licenses/by/4.0/

(c) (i) Open Access

\section{Abstract}

Aims: This study aims at designing and implementing syllabus-oriented question-bank system that is capable of producing paper-based exams with multiple forms along with answer keys. The developed software tool is named X(Chi)-Pro Milestone and supports four types of questions, namely: Multiple-choice, True/False, Short-Answer and Free-Response Essay questions. The study is motivated by the fact that student number in schools and universities is continuously growing at high, non-linear, and uncontrolled rates. This growth, however, is not accompanied by an equivalent growth of educational resources (mainly: instructors, classrooms, and labs). A direct result of this situation is having relatively large number of students in each class-room. It is observed that providing and using online-examining systems could be intractable and expensive. As an alternative, paper-based exams can be used. One main issue is that manually produced paper-based exams are of low quality because of some human factors such as instability and relatively narrow range of topics [1]. Further, it is observed that instructors usually need to spend a lot of time and energy in composing paper-based exams with multiple forms. Therefore, the use of computers for automatic production of paper-based exams from question banks is becoming more and more important. Methodology: The design and evaluation of X-Pro Milestone are done by considering a basic set of design principles that are based on a list of identified Functional and Non-Functional Requirements. Deriving those requirements is made possible by developing X-Pro Milestone using the Iterative and Incremental model from software engineering domain. Results: We demonstrate that X-Pro Milestone has a number of excellent characteristics compared to the exam-preparation and question banks tools available in market. Some of these characteristics are: ease of use and operation, user-friendly interface and good usability, high security and protection of the question bank-items, high stability, and reliability. Further, X-Pro Milestone makes initiating, maintaining and archiving Question-Banks and produced exams possible. Putting X-Pro Milestone into real use has showed that X-Pro Milestone is easy to be learned and effectively used. We demonstrate that 
$\mathrm{X}$-Pro Milestone is a cost-effective alternative to online examining systems with more and richer features and with low infrastructure requirements.

\title{
Keywords
}

\author{
Exam Preparation Tools, Syllabus-Oriented Question Banks, Partially Computerized Exams, \\ Iterative and Incremental Software Development Model, X-Pro Milestone
}

\section{Introduction}

Student numbers in universities are becoming higher every day [2] [3]. Statistics show that this increase in student numbers is not accompanied by an effective and equivalent growth of educational resources such as instructors and computer labs [4] [5]. This makes the usage of fully computerized examination tools (that allows students to set for tests and exams using a computer, usually networked) prohibitively impossible especially in developing countries where proper infrastructure is not available and not affordable [2] [3] [6].

Exams can be [5]: 1) fully computerized, 2) partially computerized, or 3) paper-based (not computerized). In fully computerized tests, each student views and directly responds to test items (or questions) using a computer. Students' responses along with the list of questions are maintained in a computer server. This makes grading exams to be fully automated [4]. In partially computerized tests, computer software is used to prepare printable exam-forms, and the student responds to exam items using a pencil. A computer software can be used later to automatically evaluate student's responses to exam items, this step can also be manual. In pure paper-based tests the student uses a pencil to respond to test items in manually prepared exam-forms. This means that computers are neither required while preparing the exam-forms nor during exam sessions. It is observed that partially computerized exams are the ideal choice when having large number of students setting for exams. In fact, under such circumstances, fully-computerized and pure paper-based tests can be prohibitively expensive [3]-[9].

In this paper we design and implement a personalized and syllabus-oriented question-bank system that is capable of producing paper-based exams with multiple forms along with answer keys. The developed software tool is named X-Pro Milestone and supports four types of questions, namely: Multiple-choice, Short-Answer, True/False and Free-Response Essay questions.

The design and evaluation of X-Pro Milestone is done by considering a basic set of design principles. Those design principles, in turn, are based on a list of identified Functional and Non-Functional Requirements. Deriving those requirements is made possible by developing X-Pro Milestone using the Iterative and Incremental model from software engineering domain.

We demonstrate that X-Pro Milestone is equipped with a number of excellent characteristics and feature that cannot be found in other exam-preparation and question-bank tools available in market. Some of these characteristics are: ease of use and operation, user-friendly Graphical User Interface (GUI) and good usability, high security and protection of the question-bankitems, high stability, and reliability. Further, X-Pro Milestone makes initiating, maintaining and archiving course syllabus question-banks and produced exam instances not only possible but also interesting. Putting X-Pro Milestone into actual use by a number of instructors has showed that X-Pro Milestone is easy to be learned and can be effectively used even by un-experienced computer users. We demonstrate that X-Pro Milestone is a cost-effective alternative to online examining systems with more attracting features.

\section{Problem Statement and Requirements Analysis}

\subsection{Problem Statement}

The following is the problem statement that we are tackling through the X-Pro Milestone software.

"Given a set of multiple-choice, true-false, short-answer, and free-response questions organized under a set of sections where each section contains a set of question pools. We would like to design a software tool that 1) randomly selects a set of $m$ question pools $P(m)$ from the set of preselected sections, and after that, 2) randomly selects a set of $m$ questions $Q(m)$ from the selected set of question pools; one question is randomly selected from 
each pool, 3) after that, the software produces a set of $n$ different exam-forms out of the set $Q(m)$.

The produced exam-forms must be optimized to minimize the effect performance interference (that is cheating) as cheating negatively affects the evaluation process and, in turn, the educational system [2] [6] [10]. Reducing cheating can be achieved through having many-enough exam-forms produced by randomly permuting 1) choice-list items (for multiple-choice questions), 2) questions, or 3) both [3]-[5]. The randomization should produce uniformly distributed correct-choices over the set of possible positions within choice-lists to reduce the negative effect of randomly responding to multiple-choice question by students."

Producing multiple exam-forms should be done by permuting 1) the list of questions, or items, this is applicable to all types of questions, including multiple-choice questions, 2) or the list of choices of each question, this applies to multiple-choices questions only, or 2) both; that is permuting questions as well as choices. The user should have the option of selecting a different set of question for each exam-form produced. Each of the $n$ examforms is to be produced along with its specific answer-key. The list of choices is to be properly permuted such that the probability of observing the correct answer at one position within the list of choices is equal to the probability of observing it at any other position (the computer uniform random number generator is to be used for that purpose). The goal of this requirement is to minimize the chance that an examiner receives high score solely by clustering his/her answers at one given position, not because of his/her real achievement.

Each question pool includes a set of questions covering a specific course objective. It is assumed that the instructor, when preparing his/her course question-bank for a given course, subdivides the syllabus into a set of well-defined course objectives or topics.

Notice that course text-books nowadays are prepared with a set of objectives for each chapter. Text-book publishers usually provide online objective question banks [11]. In fact, text-books are more and more becoming course-oriented which makes the usage of our proposed software ready for this line of book authoring.

The idea in the proposed software is to select exactly one question from each question pool. We argue that this guarantees that the produced exam effectively covers relatively wide range of topics.

The importance of the proposed software comes from the fact that exam questions in general, and multiplechoice questions specifically, are difficult to construct as they are relatively very sensitive to construction mistakes and, thus, require much of the instructor's focus [4] [12]. Studies also show that the creation of well-structured multiple-choice questions guarantees a more advanced understanding of the relationships between the concepts of the learning material as presented in the textbook [8] [9]. We believe that helping instructors to establish and maintain course question banks is vitally needed to maintain, refine, improve and reuse high-quality well-constructed exam questions.

\subsection{Requirement-Analysis of X-Pro Milestone}

In addition to allowing instructors to create, maintain and produce course question banks, the following list represents the functional and non-functional requirements considered when developing the X-Pro Milestone software [13] [14]:

Backup and Recovery: The application should allow for backing up and archiving course question banks for future referencing.

Documentation: The application's user interface should be user-friendly and intuitive and thus require minor documentation. A user should be able to easily learn how to use the software without the need to always refer to a lengthy and difficult-to-memorize documentation.

Efficiency: Defined as resource consumption for given load. The application should be simple and not need more than a normal desktop computer with no specialized hardware, network or software components. The application should also be stand alone and require no other software.

Effectiveness (resulting performance in relation to effort): Producing exams using the software should take minimal effort from the instructor. This, however, should not negatively affect the functionality of the application.

Emotional factors: In addition to having a simple and intuitive user-friendly user interface, the application should give the instructor positive feelings toward the value of the software. In fact, those who used the software already are pleased and feel comfortable as the application saved them long time and efforts. The true value of the application is in the fact that the effort an instructor put when preparing an exam years ago contributes to the success and the quality of the exam the instructor will be producing next. Test items that were added to the 
course question banks should be available to be included in future exam instances. We refer to this as test-item reusability.

Exploitability and Privacy: Course question banks are meant to be private and personal. That's why our application should store and maintain those question banks on the user's local machine which is supposed to be personal and not accessible by unwanted users.

Extensibility: In software engineering, software versioning is used to control software versions and updates. The application should be produced in versions such that required updates are deployed to its installations instantly. For that, the application should check for updates each time the instructor runs it. In addition to that, the course question-bank is stored along with the version number of the software. This allows the user to use future versions of the software and migrate his/her course question banks to the newer version and benefit from the added features, and to carry-forward his/her customizations to the next major version upgrade. This is referred to backward compatibility of software. To achieve that, the application should follow open and widely accepted standards.

Failure Management: the application should help instructor find the location of each question in the produced exam-form and preview it in the question bank. This should help the instructor fix any question that may have typos or to continuously improve the quality and readability of the questions (check next point).

Maintainability, Modifiability and Quality: The application should allow for incremental improvement of course question banks. Specific questions must be properly referenced so that the user can revisit previously saved and archived questions for editing and quality improvement. This should allow for having incremental improvement of test items.

Portability: Course question banks need to be stored in a way that allows the user to copy or move them around (if needed).

Scalability (Horizontal, Vertical): In our context, horizontal scalability refers to the ability to add sections and question pools to a given course bank. Vertical scalability refers to the capability of course question-bank system to allow the user to add questions to a specific question pool (that represents a specific course learning objective). The X-Pro Milestone should provide both features.

Security and Protection: The X-Pro Milestone is meant to be personal and to be installed on the user's personal machine. Thus, the security feature depends solely on how secure the file system of the user's desktop is. The protection feature also depends solely on how the user's account on the host machine is protected.

The above requirements have been obtained through putting two previous versions of similar application into service since year 2001. Some features provided by X-Pro Milestone may be needed by specific type of users. But for completeness, we included every feature we found to be useful to any group of users. Deriving those requirements is made possible by developing X-Pro Milestone using the Iterative and incremental development approach from software engineering domain (Figure 1).

The Iterative and incremental development approach involves testing and evaluation while development, this allows for fine modification and early improvement of the initial design of software while implementing it before the deployment step. Many requirements of the software, however, were collected after software deployment by taking feedbacks from real users. Those requirements were collected since 2001 when the first version of the software was put into service [3]-[5].

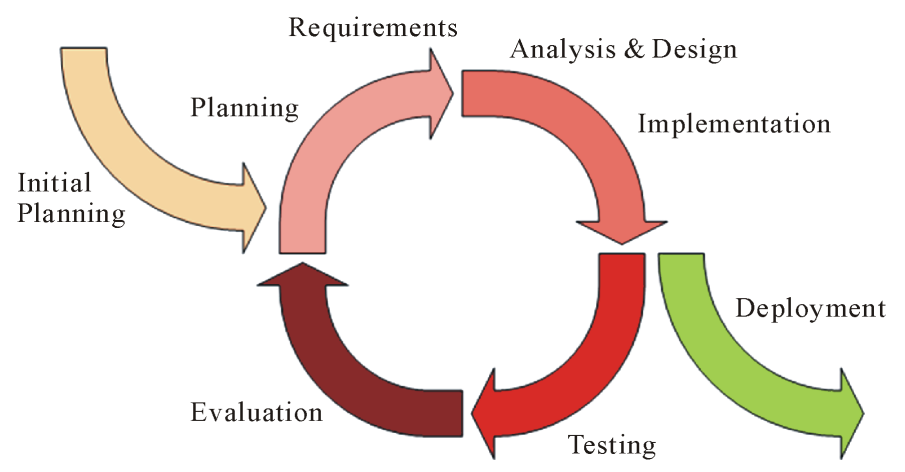

Figure 1. The Iterative and incremental development approach from software engineering domain. 
The key idea behind this model is to develop the targeted software through repeated cycles (i.e., iterative) and in smaller portions at a time (i.e., incremental) as shown in Figure 1. This allows software developers to benefit from what they learned so far during development of earlier parts or revisions of the software. Learning comes from both 1) the development and 2) practical use of the system.

Historically, X-Pro Milestone has started in 1999 with a simple implementation of the software that simply allows the user to enter multiple-choice question, the software produces printable forms of automatically produced set of exam instances. The software requirements are enriched since then and the software is iteratively enhanced until X-Pro Milestone is implemented with more features [15]. At each iteration, design modifications are made and new functional capabilities are added to that simple implementation.

\subsection{Functional Requirements of X-Pro Milestone}

Next we summarize a set of functional requirements that we considered when developing X-pro Milestone. We also describe how our software meets those requirements.

Management of Course Question Banks: This includes creating and maintaining course question banks. The user is also able to archive and create backup copies of those course question banks.

Support of wide and comprehensive types of questions: The developed application supports wide spectrum of question types: multiple-choice, true/false, short-answer and free-response (essay) questions.

Allowing the User to Include Figures within Questions: X-Pro Milestone allows the user to add figures in the form of 1) images (of any format that is supported by web browsers; e.g., JPG, PNG, and GIF formats), 2) HTML code snippets, and 3) plain text contents. The figures can be inserted within any question directly. Alternatively, the user may include the figure at the end of the exam instance and the application will automatically add an appropriate reference to that figure within the question text. Figures in exam instances are automatically assigned unique ID numbers; the user needs not to be worried about numbering at all.

Next we list some extra features added to X-Pro Milestone. Through putting the application into real service, we found that those features prove useful to some users:

- Allowing users to add comment lines within question banks. Those lines are ignored when producing exam instances.

- Properly adding indentation and numbering to the course question-bank sections and to the question pools. This improves the readability of the question bank. It should also allow the user to quickly navigate to any required section or pool.

- Automatic building of a navigation tree with foldable and expandable nodes that should allow the user to preview the structure of the current course question-bank and directly navigate to any section or question pool within the course bank.

- Provide a mechanism for the user to label sections and question pools.

- Allowing the user to add text formatting tags in questions. One possible benefit of this feature is helping the user emphasize certain keywords in the question to bring the attention of the student to them. This also should help adding equations and probably computer code segments within the question text.

\section{The Proposed Software}

In this section we describe our software, the X-Pro Milestone, and show how we could successfully achieve the requirements listed in the previous section.

\subsection{Understanding the X-Pro Milestone Input}

X-Pro Milestone Supports a number of question types. Those are, Multiple-Choice, Short-Answer, True-False and Free-Response question types.

Each type of the three questions has the same generic structure. Figure 2(a) shows the basic structure of the course question-bank in X-Pro Milestone. That is; a number of sections, each section is composed of a number of question pools. Each question pool, in turn, includes one or more question. A question pool represents a specific and well-defined course objective or subtopic. This means that questions that belong to the same question pool are somehow topically similar and cannot be included in the same exam-form instance. The wisdom behind this course question-bank structuring is to guarantee that produced exams properly cover the course material. 


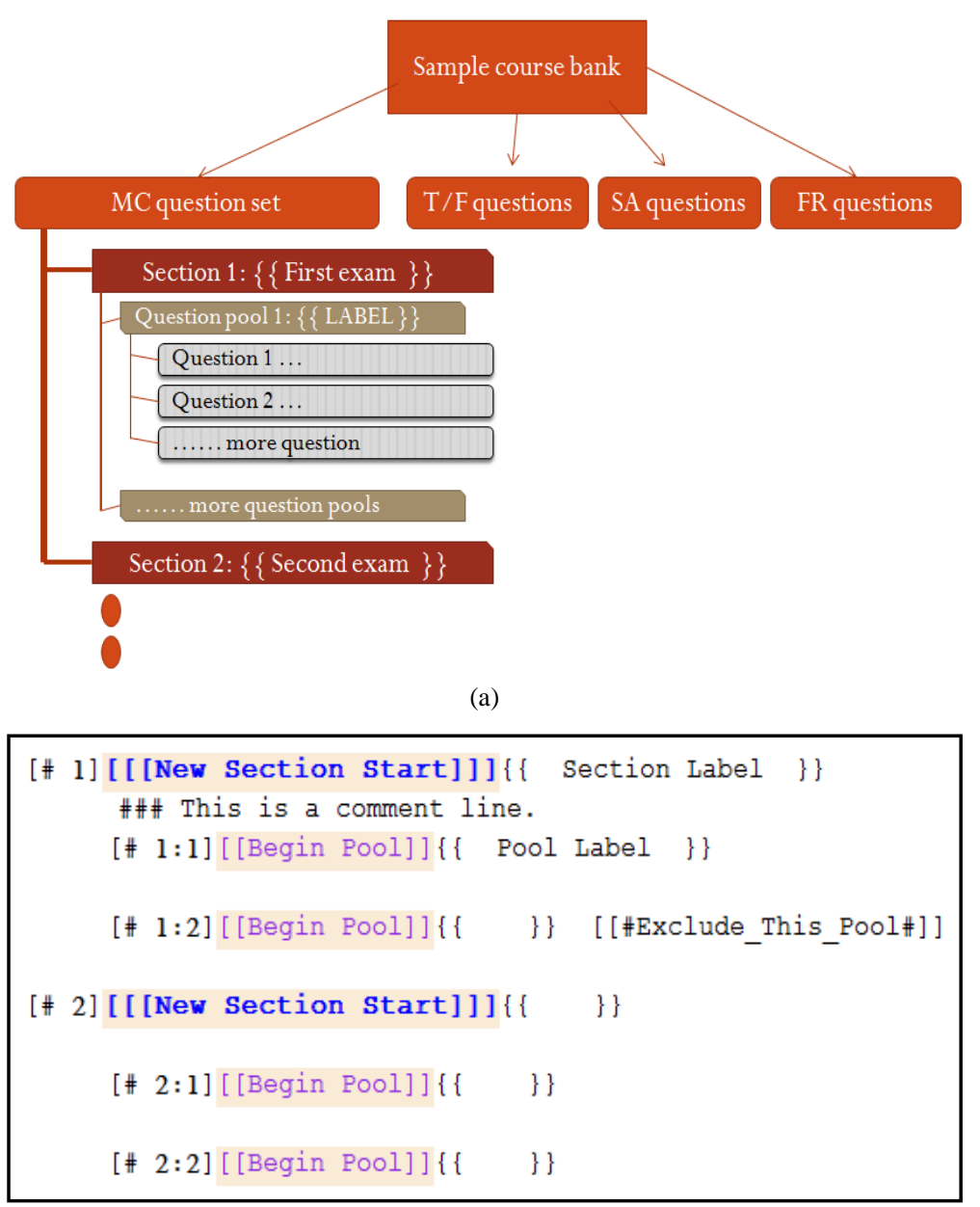

(b)

Figure 2. (a) X-Pro Milestone course question-bank structure; (b) X-Pro Milestone Sample course question-bank structure. tions.

The following is a list of the basic X-Pro Milestone tags used to indicate sections, question pools and ques-

- The [[New Section Start]] starts a new section.

- The [[Begin Pool]] tag starts a new pool.

- The [[Begin Question]] tag starts a new question.

Figure 2(b) shows a sample of two sections. Each section is composed of two question pools. The section label can be something like $\{\{$ First Exam Set $\}$ or $\{\{$ Final Exam Set $\}$. The pool label describes the specific course objective being measured by the list of questions enclosed within the current pool. As a good practice, we recommend that the instructor subdivides the course objectives into three sections. The first section represents the set of objectives to be covered and evaluated in the first midterm exam. The second section represents the set of objectives to be covered and evaluated in the second midterm exam. And finally the last section represents the set of objectives to be covered and evaluated after the second exam and before the final exam. Later, when producing exam instances, one question is to be randomly selected from each pool of a given section. This way, the instructor guarantees that the same question will not appear twice in the exam and that the list of selected questions covers the set of course objectives properly.

The number of pools inside each section is left to the instructor. However, as a good practice, we recommend that the number of pools in the "First Exam" section be equal to 20 if the weight of the first midterm exam is 20 points. In this case, each question (that will be selected from one of the 20 pools available) will be of one point weight. Same applies to the "Second Exam" section. The "Final Exam" section is quite different. It is known 
that final exams cover 1) the topics included in the first and the second midterm exams, in addition to 2) the material covered after the second exam and before the final exam date. This means that the set of questions that will appear in the final exam will be selected from the set of pools that are located in the first, the second and the last section of the course question bank. Consequently, the instructor is free to subdivide the "final exam" section into any number of pools he/she prefers.

In X-Pro Milestone, lines that begins with the "Comment line” tag (that is “\#\#”) are considered comment lines and will be skipped while processing the user's input (as shown in Figure 2(b)).

\subsection{X-Pro Milestone Syntax for Supported Question Types}

Figure 3 shows a sample multiple-choice question. The figure shows two sections each is of two question pools.

The [[Begin Question]] indicates the beginning of a new multiple-choice question. The [[Begin Choice List]] tag indicates that the lines after are the list of choices of the current question. The set of lines after the [[Begin Question]] and before the [[Begin Choice List]] tags represent the multiple-choice question stem. This means that the question stem may be composed of multiple lines. However, the [[Begin Choice List]] tag is optional. In this case the question stem is assumed to be composed of one single line. The lines that follow that line represent the list of choices.

Each line in the list of choices represents one choice. A choice my not span multiple lines. The "<ca>" next to a choice indicates that this choice is a correct choice (used later by the application to produce the answer key table). The $<\mathrm{ca}>$ is referred to as the "correct answer" tag. A multiple-choice question may have one or more correct choice.

Each multiple-choice question can have up to six choices. Those choices will be later permuted to produce exam-forms. To force one specific choice to remain in-place (will not be permuted), you put the "do not permute" tag next to that choice; that is the $<\mathrm{dnp}>$ tag. This helps the user to have choices like "All above" or "both $A$ and $C$ are correct" choices.

The [[\#Inline_Choices\#]]\} tag next to the [[Begin Question]] tag indicates that the list of choice in the produced exam-form will be put in-line (next to each other) as shown in Figure 4. Otherwise, they will be viewed in a normal vertical list as shown in Figure 5.

Figure 6 shows a sample of two true-false questions and two short-answer questions. Each question appears in a single line. Each question is of two parts: 1) the question text, and 2) the correct answer put between the "[[" and "]]" tags (referred to as the short-answer opening and closing tags).

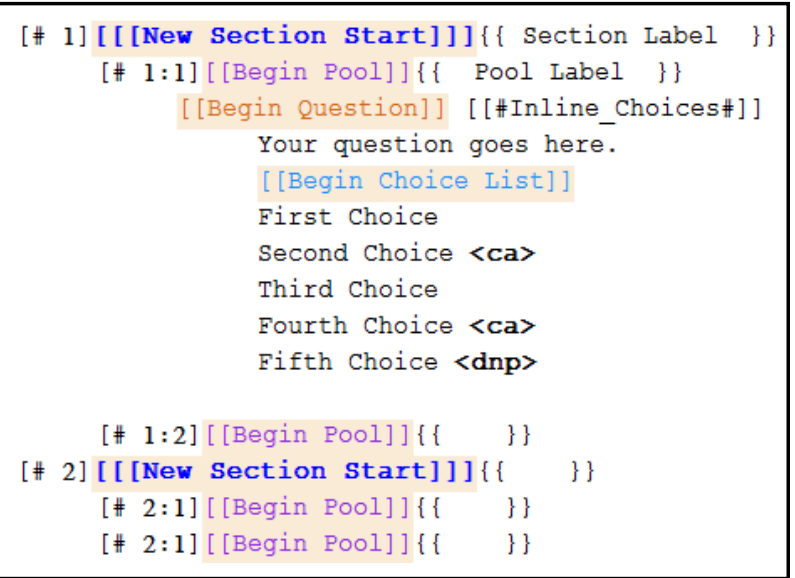

Figure 3. The syntax of a typical multiple-choice question in X-Pro Milestone.

1. Your question goes here.

A. First Choice B. Second Choice C. Third Choice D. Fourth Choice E. Fifth Choice

Figure 4. A sample multiple-choice question with its choice-list displayed inline. 
Figure 7 shows a sample free-response essay question. Each free-response essay question is of two parts: 1) the question text that may span multiple lines. It appears after the [[Begin Question]] tag and before the [[Begin answer]] tag, and 2) the correct answer that will appear after the [[Begin Answer]] tag. Again, the answer may also span multiple lines.

\subsection{X-Pro Milestone User's Interface}

Figure 8 shows a screen shot for the main user interface of the X-Pro Milestone. It shows five areas; the menu bar, the toolbar, the left pane and the right pane, and finally the Application Flags areas.

The left pane displays the basic functional tab pages of X-Pro Milestone. The key tab pages are:

- The "Multiple-choice questions" tab page: through which the user enters the set of Multiple-Choice (MC) questions.

- The "Short-Answer questions" tab page: through which the user enters the set of Short-Answer (SA) questions.

- The "True-False questions" tab page: through which the user enters the set of True/False (TF) questions.

- The "Free-Response questions" tab page: through which the user enters the set of Free-Response essay (FR) questions.

Figure 9 shows the "Multiple-choice questions" tab page. The pane on the left provides a space in which the user enters the multiple-choice questions. The pane at the middle is the current "Tab Page Explorer" that allows the user to navigate directly to any of the sections and pools available in the current tab page.

Figure 10 shows the "Figures and attachments" tab page through which the user manipulates the set of figures referred to within the exam questions (this includes: importing, removing, previewing, and adding notes to any figure in the course bank). Each figure file must have a unique label of the user's choice (otherwise, the software will automatically assign a unique label to the figure while importing it). This label is included in the filename of the figure file between double square brackets. This label will be later used when inserting figures inside exam questions. This tab page displays information about the selected figure from the list, e.g., its full filename,

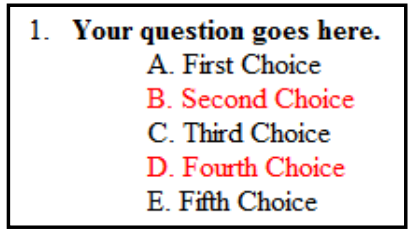

Figure 5. A sample multiple-choice question with its choice-list displayed in multiple lines.

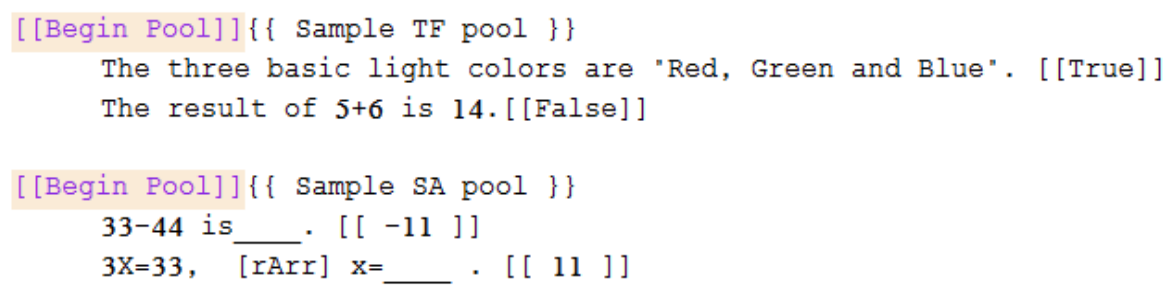

Figure 6. Sample True-False questions.

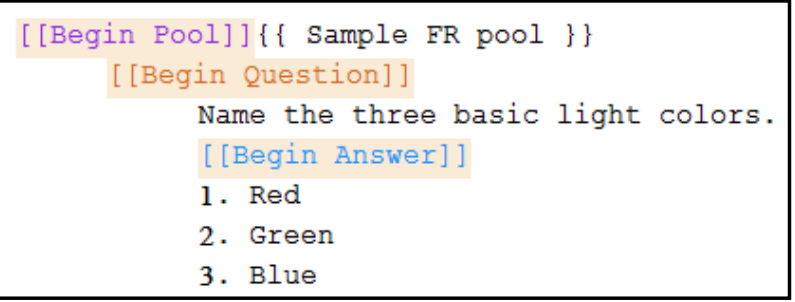




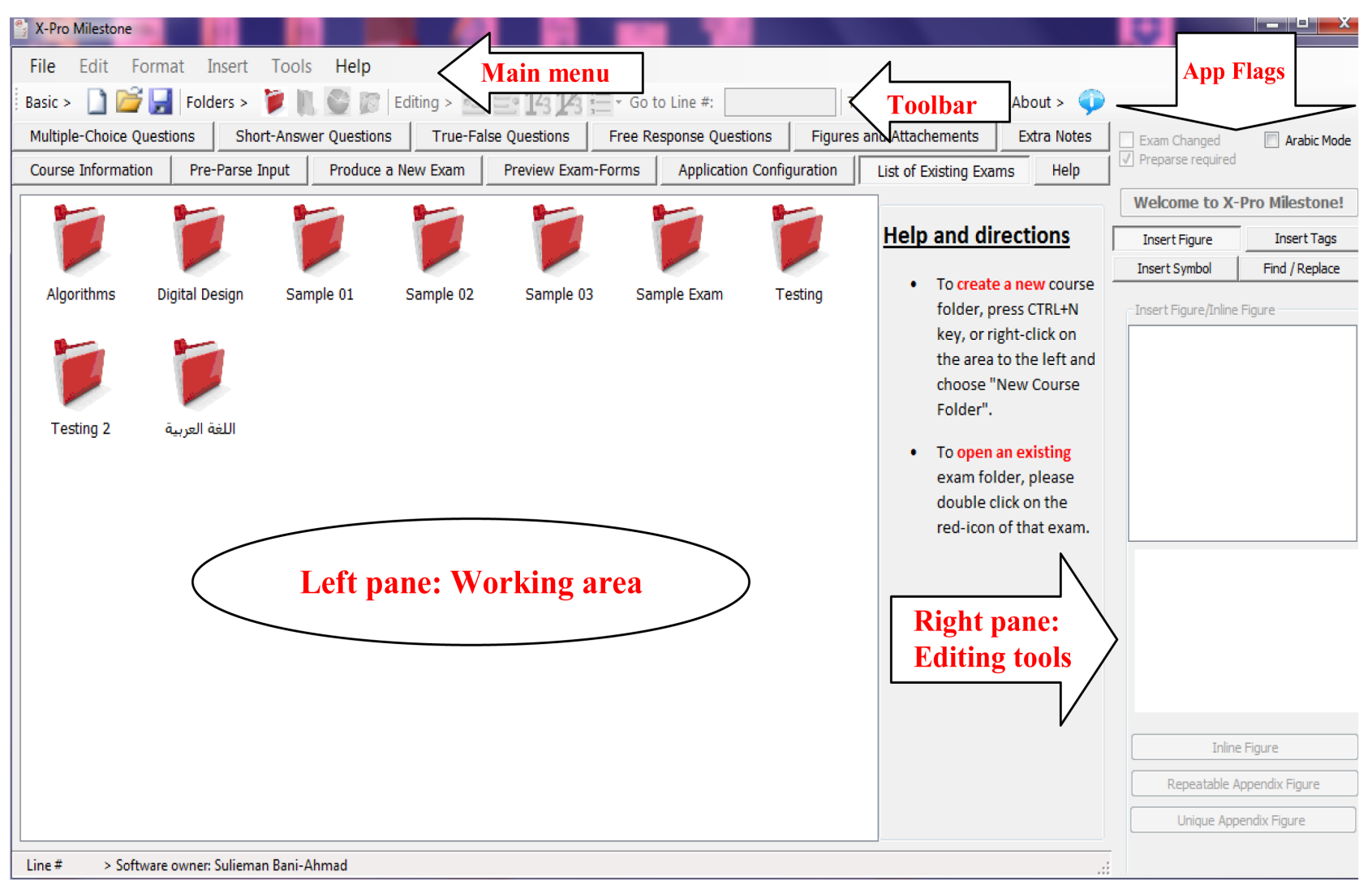

Figure 8. Major parts of the X-Pro Milestone user interface.

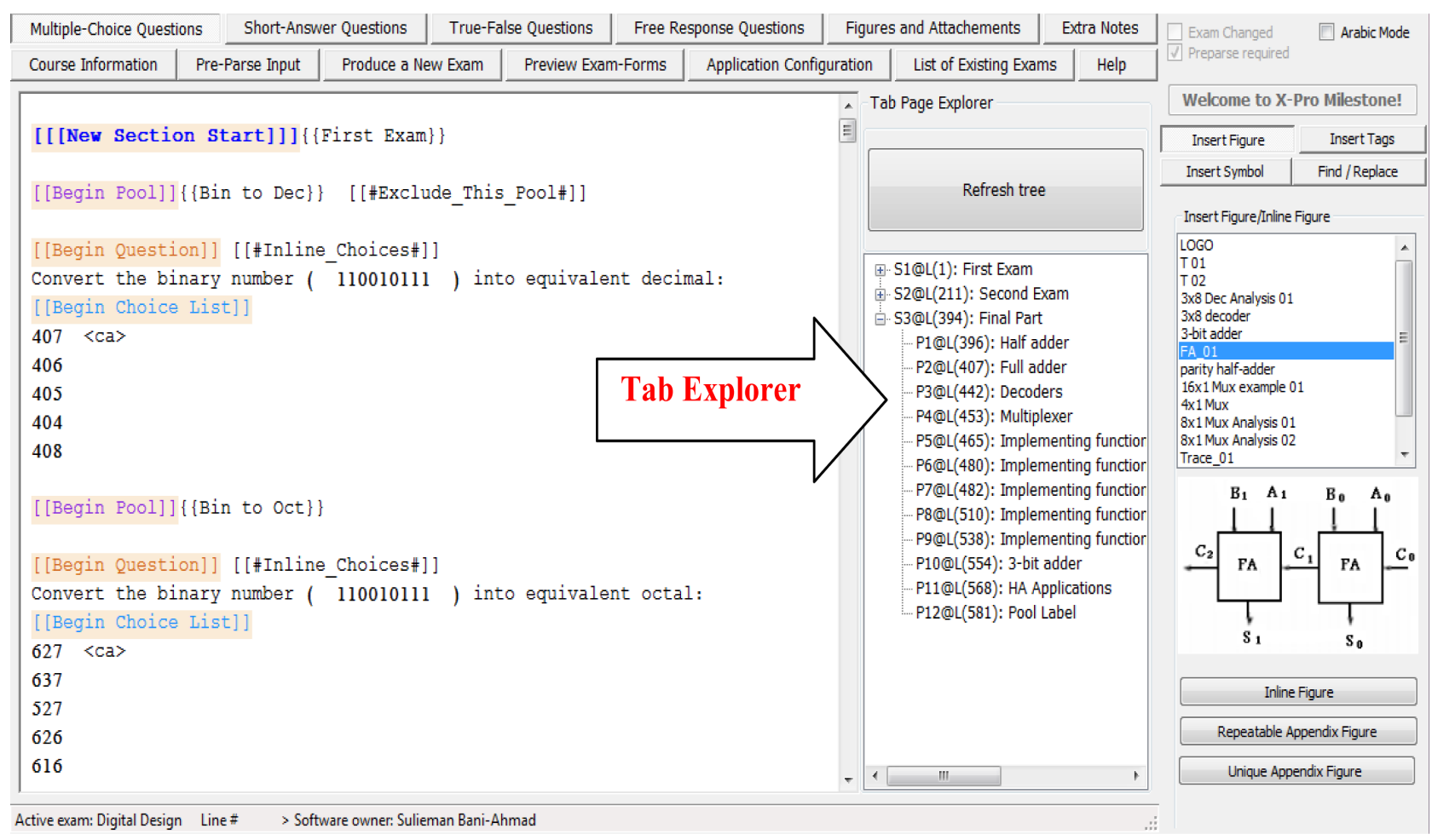

Figure 9. The “Multiple-choice questions” tab page. 


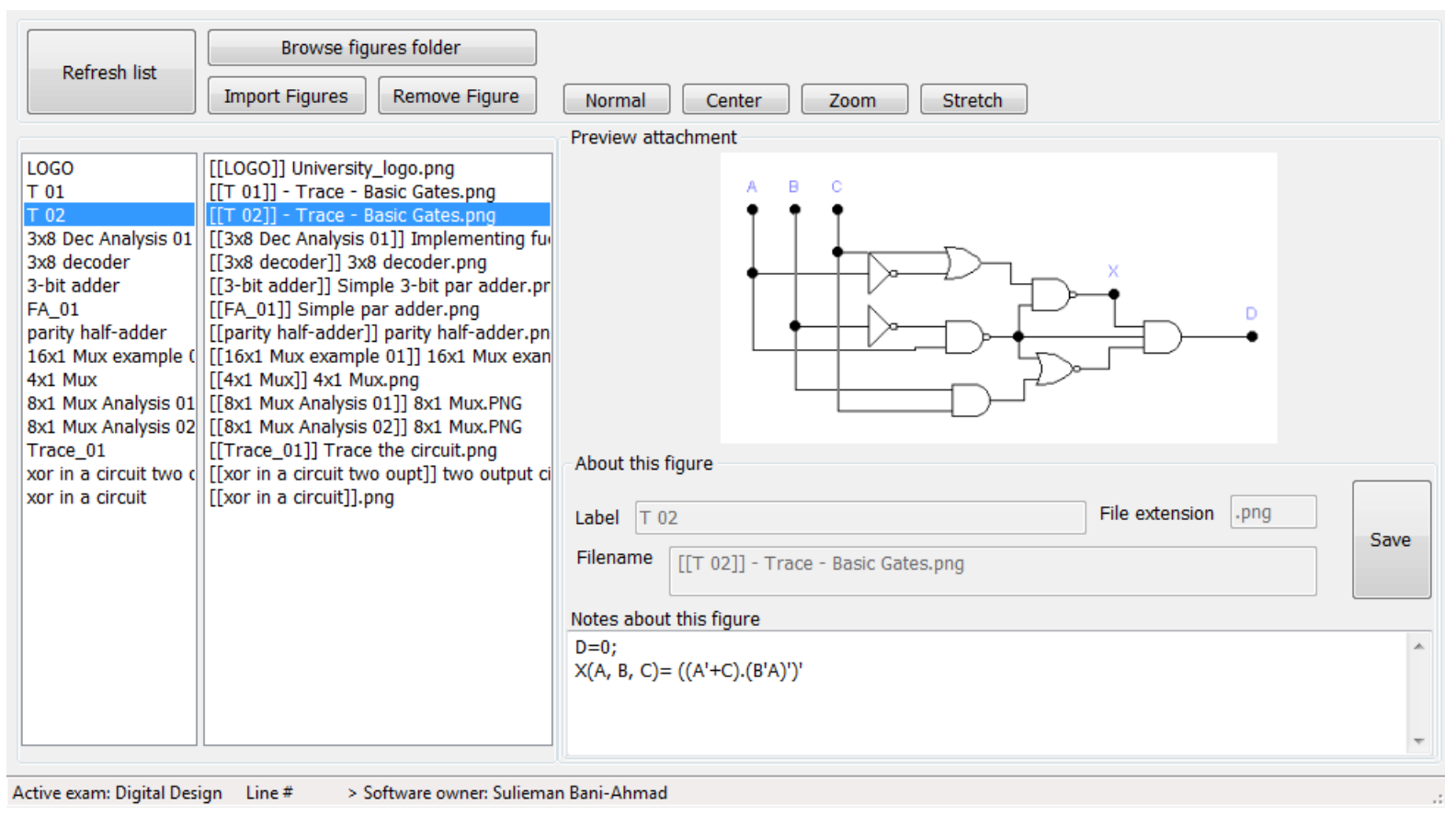

Figure 10. The "Figures and attachments" tab page.

file extension, label, notes about this specific figure, and it shows the user a preview of this figure.

\subsection{Producing Exam Instances Using X-Pro Milestone}

In order to produce an exam out of an existing course question-bank, the following steps are to be taken:

Step A: Pre-parsing the course question-bank using through the "Pre-Parse" tool located at the "PreParse Input” tab page (Figure 11)

Before the user can produce exam instances from his/her course question bank, he/she needs to pre-parse the course question-bank to check if the course has syntax or structural errors. If the user chooses to check the "Exclude empty pools" check box shown in Figure 11, then observing empty pools will be considered as warnings not as errors.

Other errors that the pre-parser may identify are 1) finding empty sections, 2) finding a multiple choice question with no choice list or only one single choice item.

Step B: Producing Exam instances through the "Produce a New Exam” tab page.

After the user pre-pares the course question-bank with no errors, the "Produce a New Exam" tab page will be activated and its content will be refreshed. The user can now produce exam instances. This is done in four steps.

Step B.1: In which the user chooses the set of sections to be included and the number of pools to be randomly selected (Figure 12).

Step B.2: Setting exam information: As shown in Figure 13. This includes for example the exam date, time and date, the exam class (first, second or final exam), etc....

Step B.3: Setting exam instructions: as shown in Figure 14.

Step B.4: Choosing the form generator settings through the "Produce a New Exam" tab page as shown in Figure 15. Those settings include: 1) the number of forms required, 2) the form ID style, 3) wither the user wants to hide the form ID from the student in the produced forms, 4) the permutation options, 5) wither the user wants to have the same set of questions in all produced forms or not, 6) the multiple-choice answer table and answer-key styles.

Step B.5: Previewing produced exam-forms using the "Preview and Export Exam-Forms" tab page (Figure 16).

The user can now preview the produced exam-forms. The user can choose to preview the produced examform using Microsoft Internet Explorer (recommended) or Google Chrome. 


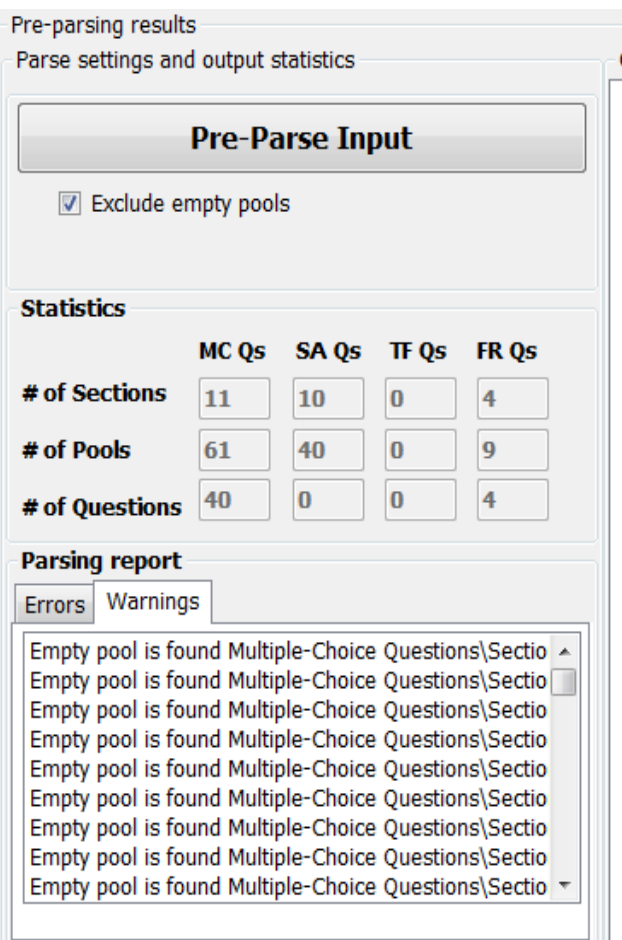

Course Tree

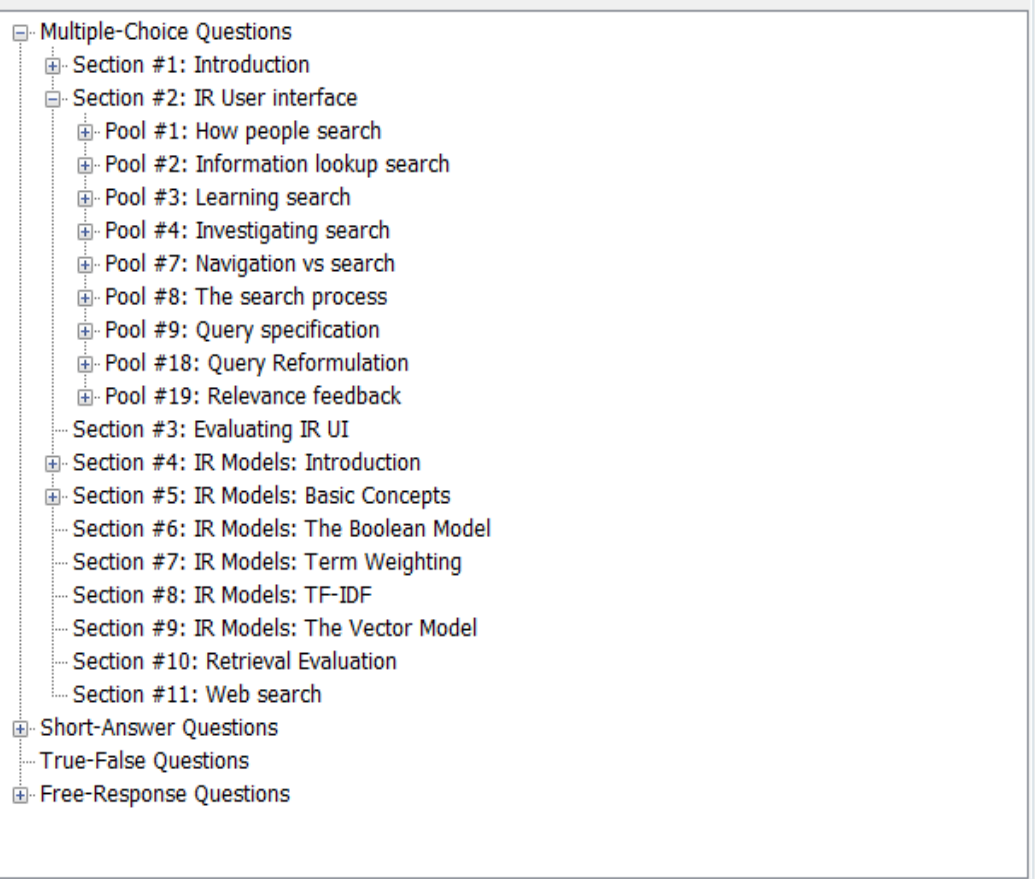

Figure 11. The "Pre-Parse Input” tab page.

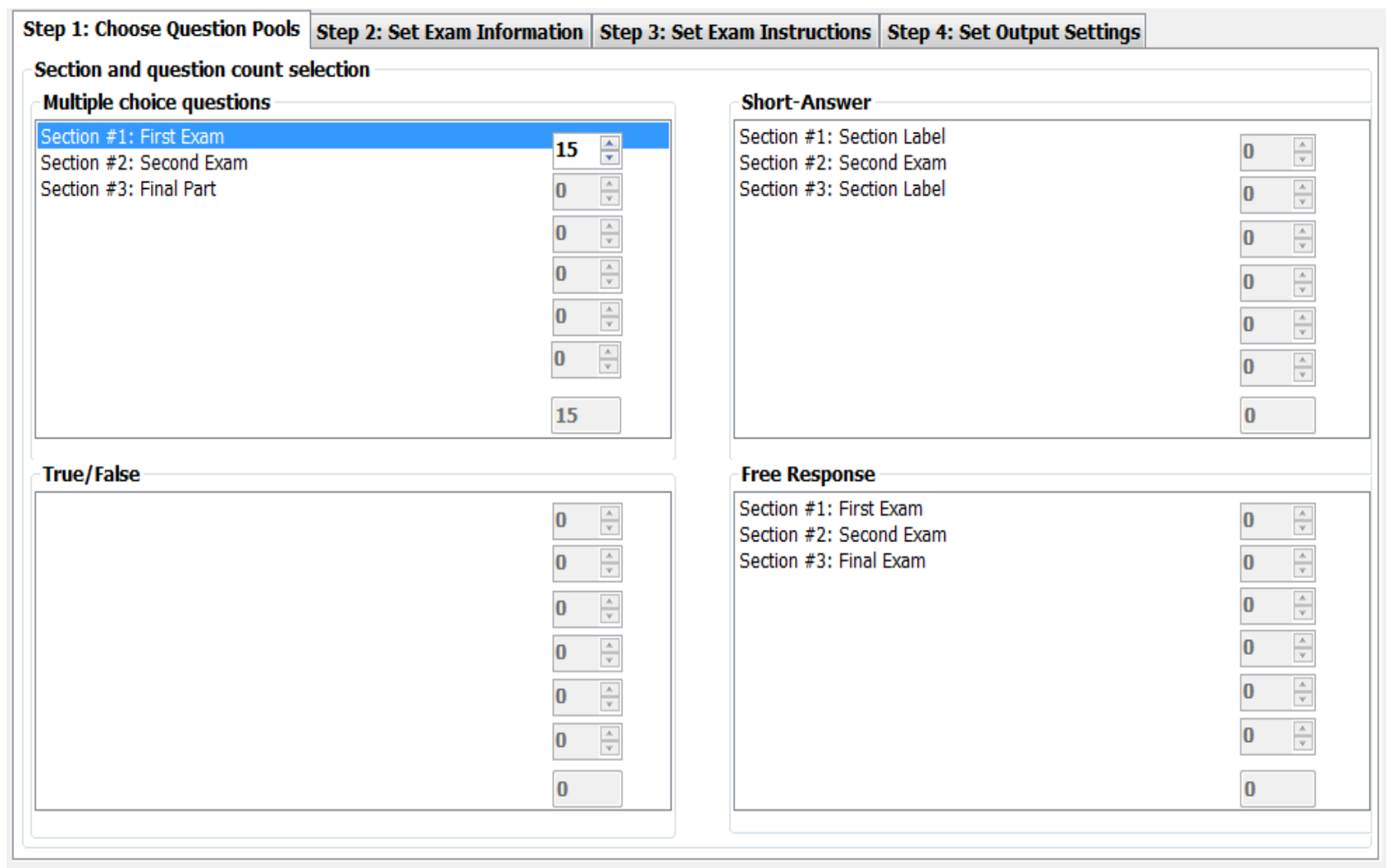

Figure 12. Choosing sections and question pools for exam production. 


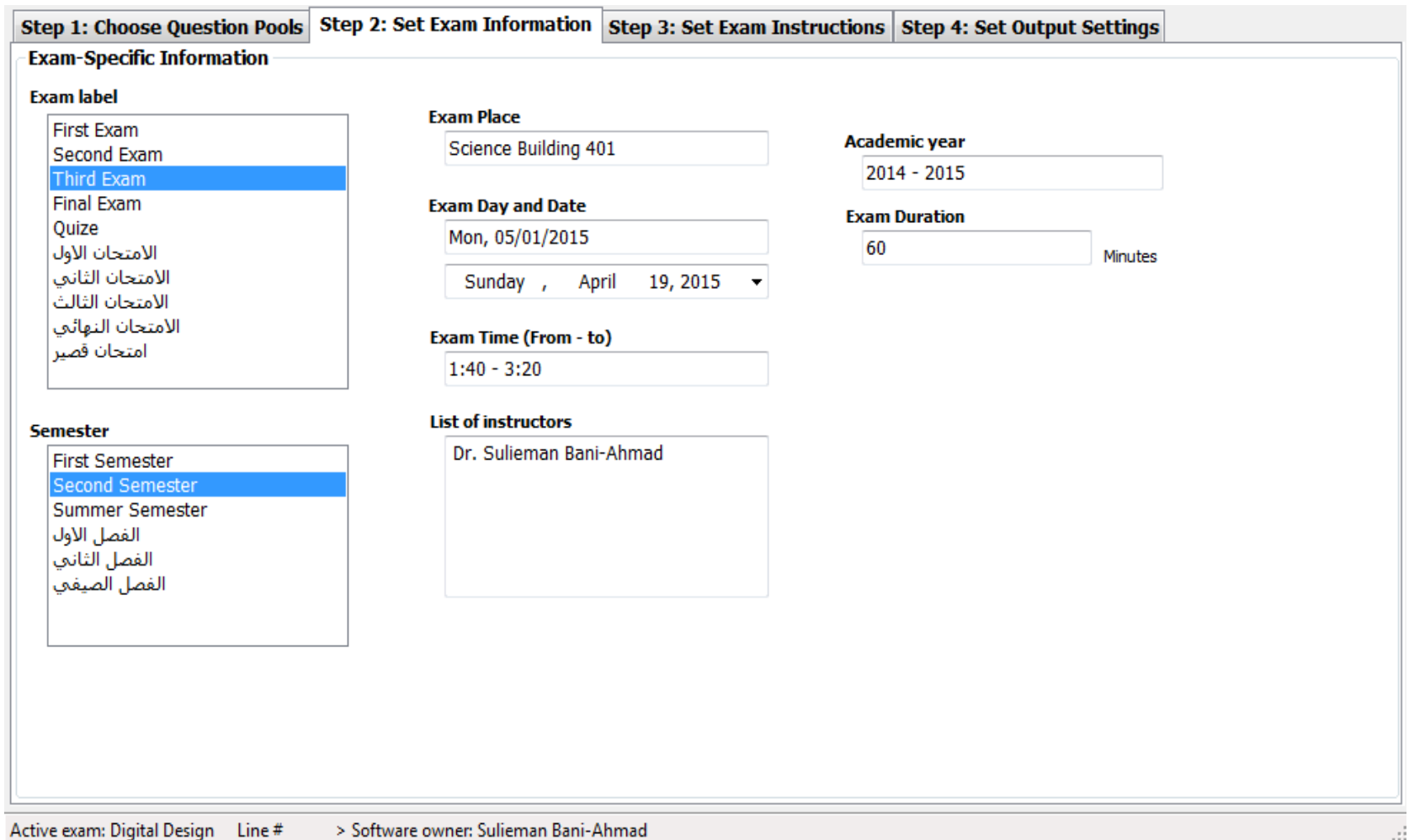

Figure 13. Setting exam information for exam production.

\section{\begin{tabular}{|l|l|l|l|l|l|}
\hline Step 1: Choose Question Pools & Step 2: Set Exam Information Step 3: Set Exam Instructions & Step 4: Set Output Settings
\end{tabular}}

Choose all what apply to this exam please.

$\checkmark$ Calculators are NIOT allowed.

$\square$ Calculators are allowed.

$\square$ Place your answers in the answer table(s).

$\checkmark$ Turn Your mobiles off please.

$\square$ Circle you instructor name from the list.

Additional exam instructions can be added next ...

Place your answers in the answer table(s).
Place your answers in the answer table(s).

Calculators are NOT allowed.

Turn Your mobiles OFF please.

Figure 14. Setting exam instructions for exam production. 


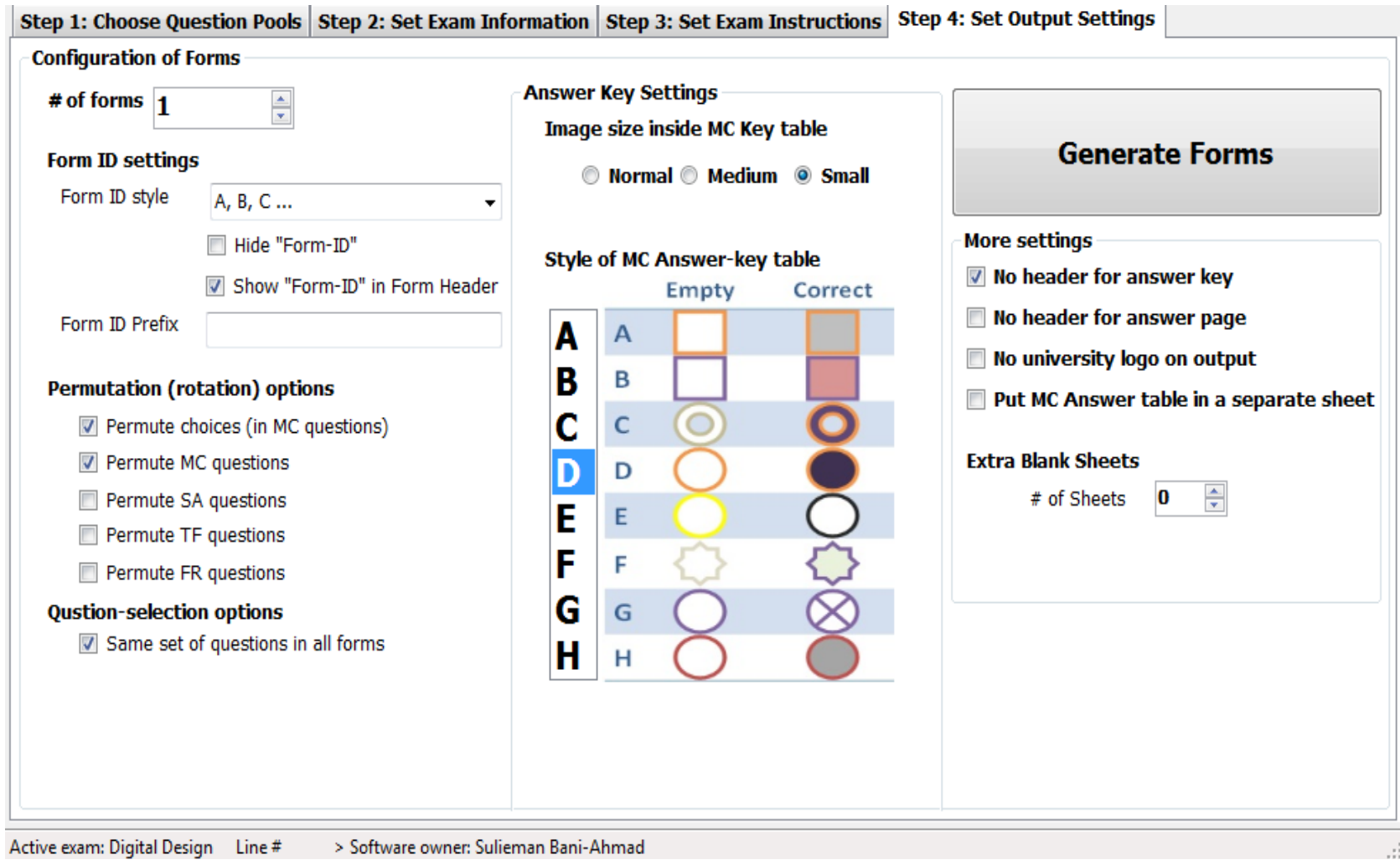

Figure 15. Choosing form-generator setting for exam production.

\begin{tabular}{|c|c|c|c|}
\hline Course Information & Pre-Parse Input & Produce a New Exam & Preview Exam-Forms \\
\hline \multicolumn{4}{|l|}{ Student name: } \\
\hline \multicolumn{4}{|c|}{$\begin{array}{l}\text { Al-Balqa Applied University, Information Technology, Computer Information Systems } \\
\text { Digital Logic Design: Third Exam, Second Semester } 2014-2015 \\
\text { Exam Day. Date. Time and Place: Mon, 05/01/2015, 1:40 - 3:20, Science Building 401.: [.A] }\end{array}$} \\
\hline
\end{tabular}

\section{Multiple-Choice Questions}

\begin{tabular}{|c|c|c|c|c|c|c|c|c|c|c|c|c|c|c|}
\hline \multirow{2}{*}{} & $\mathbf{A}$ & $\mathbf{B}$ & $\mathbf{C}$ & $\mathbf{D}$ & $\mathbf{E}$ & $\mathbf{F}$ & & $\boldsymbol{*}$ & $\mathbf{A}$ & $\mathbf{B}$ & $\mathbf{C}$ & $\mathbf{D}$ & $\mathbf{E}$ & $\mathbf{F}$ \\
\hline $\mathbf{1}$ & $\bigcirc$ & $\bigcirc$ & $\bigcirc$ & $\bigcirc$ & $\bigcirc$ & $\bigcirc$ & & $\mathbf{9}$ & $\bigcirc$ & $\bigcirc$ & $\bigcirc$ & $\bigcirc$ & $\bigcirc$ & $\bigcirc$ \\
\hline $\mathbf{2}$ & $\bigcirc$ & $\bigcirc$ & $\bigcirc$ & $\bigcirc$ & $\bigcirc$ & $\bigcirc$ & & $\mathbf{1 0}$ & $\bigcirc$ & $\bigcirc$ & $\bigcirc$ & $\bigcirc$ & $\bigcirc$ & $\bigcirc$ \\
\hline $\mathbf{3}$ & $\bigcirc$ & $\bigcirc$ & $\bigcirc$ & $\bigcirc$ & $\bigcirc$ & $\bigcirc$ & & $\mathbf{1 1}$ & $\bigcirc$ & $\bigcirc$ & $\bigcirc$ & $\bigcirc$ & $\bigcirc$ & $\bigcirc$ \\
\hline $\mathbf{4}$ & $\bigcirc$ & $\bigcirc$ & $\bigcirc$ & $\bigcirc$ & $\bigcirc$ & $\bigcirc$ & & $\mathbf{1 2}$ & $\bigcirc$ & $\bigcirc$ & $\bigcirc$ & $\bigcirc$ & $\bigcirc$ & $\bigcirc$ \\
\hline $\mathbf{5}$ & $\bigcirc$ & $\bigcirc$ & $\bigcirc$ & $\bigcirc$ & $\bigcirc$ & $\bigcirc$ & & $\mathbf{1 3}$ & $\bigcirc$ & $\bigcirc$ & $\bigcirc$ & $\bigcirc$ & $\bigcirc$ & $\bigcirc$ \\
\hline $\mathbf{6}$ & $\bigcirc$ & $\bigcirc$ & $\bigcirc$ & $\bigcirc$ & $\bigcirc$ & $\bigcirc$ & & $\mathbf{1 4}$ & $\bigcirc$ & $\bigcirc$ & $\bigcirc$ & $\bigcirc$ & $\bigcirc$ & $\bigcirc$ \\
\hline $\mathbf{7}$ & $\bigcirc$ & $\bigcirc$ & $\bigcirc$ & $\bigcirc$ & $\bigcirc$ & $\bigcirc$ & & $\mathbf{1 5}$ & $\bigcirc$ & $\bigcirc$ & $\bigcirc$ & $\bigcirc$ & $\bigcirc$ & $\bigcirc$ \\
\hline $\mathbf{8}$ & $\bigcirc$ & $\bigcirc$ & $\bigcirc$ & $\bigcirc$ & $\bigcirc$ & $\bigcirc$ & & & & & & & & \\
\hline
\end{tabular}
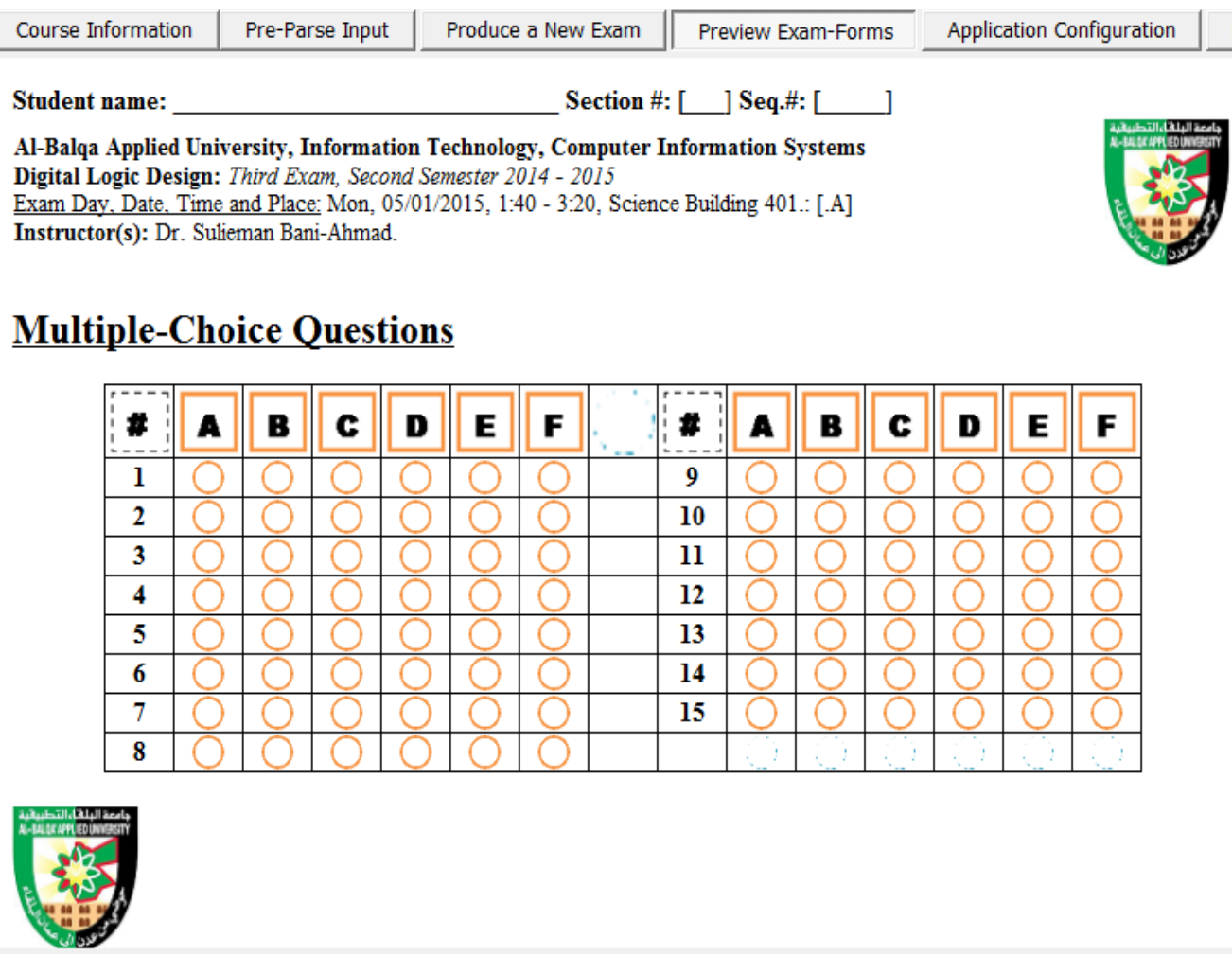
List of Existing Exams

Figure 16. Previewing produced exam-forms. 


\subsection{Printouts of Exam Instances}

In order to have a printed copy of the form, the user needs to preview the exam-form using Microsoft Internet Explorer (or any available up-to-date browser) and print it out through the browser.

Figure 17 shows a sample multiple-choice questions answer key. The user may print this table on a thermal slide to facilitate student answer table grading.

\subsection{Extra Features of X-Pro Milestone}

Next are some of the extra features that X-Pro Milestone provides.

\subsubsection{Manipulation of Course Question Banks}

Through the "List of Existing Exams" tab page (Figure 18), the user can 1) create a new course-bank, 2) open existing course question banks (for editing), 3) backup or archive an already existing one.

\subsubsection{Inserting Figure from the Pre-Fed Set of Figures}

Figures can be inserted inside the text of any question (and even in the choice list of a multiple-choice question). Figures inside the course question-bank have one of three settings:

1) Inline Figures

A figure can be inserted inline; that is, it will appear with the question in the produced exam-form (i.e., it will not appear at the end of the exam-form as appendix as illustrated in Figure 19).

\section{2) Repeatable Appendix Figures}

A figure can be inserted as a repeatable figure at the end of the exam-form as appendix. That is; if the figure is referenced inside $\boldsymbol{n}$ questions in the same exam-form, then the same figure will appear $\boldsymbol{n}$ time in the appendix of the exam-form. Notice that the figure inside the question will be replaced by a proper reference to the

\begin{tabular}{|c|c|c|c|c|c|c|c|c|c|c|c|c|c|c|c|}
\hline \multicolumn{2}{|c|}{ Course Information } & \multicolumn{3}{|c|}{ Pre-Parse Input } & \multicolumn{3}{|c|}{ Produce a New Exam } & \multicolumn{4}{|c|}{ Preview Exam-Forms } & \multicolumn{3}{|c|}{ Application Configuration } & List 0 \\
\hline \multicolumn{15}{|c|}{ 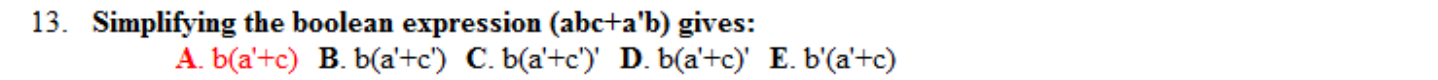 } & ^A \\
\hline \multicolumn{16}{|c|}{$\begin{array}{l}\text { 14. Convert the number (555) })_{\text {Dec }} \text { into equivalent octal: } \\
\begin{array}{lllll}\text { A. } 2553 & \text { B. } 1053 & \text { C. } 2053 & \text { D. } 1835 & \text { E. } 2253\end{array}\end{array}$} \\
\hline \multicolumn{16}{|c|}{$\begin{array}{l}\text { 15. Convert the binary number ( } 110010111 \text { ) into equivalent decimal: } \\
\begin{array}{lllll}\text { A. } 407 & \text { B. } 404 & \text { C. } 405 & \text { D. } 406 & \text { E. } 408\end{array}\end{array}$} \\
\hline \multicolumn{16}{|c|}{ Multiple-Choice Questions } \\
\hline & & $\mathbf{B}$ & $\mathbf{6}$ & D & $\mathbf{E}$ & $\mathbf{F}$ & & $\#$ & $\mathbf{A}$ & $\mathbf{B}$ & $\mathbf{6}$ & D & $\mathbf{E}$ & $\mathbf{F}$ & \\
\hline 1 & & & O & O & O & O & & 9 & O & O & O & $\mathrm{O}$ & O & & \\
\hline 2 & & 8 & O & & 0 & O & & 10 & O & 0 & O & & 0 & 2 & \\
\hline 3 & & & O & & 0 & 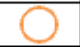 & & 11 & O & O & & D & 0 & $\overline{5}$ & \\
\hline 4 & & & 0 & & 0 & $C$ & & 12 & 0 & 0 & 0 & 0 & 0 & & 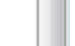 \\
\hline 5 & & & 0 & & & & & 13 & 0 & 0 & 0 & 0 & 0 & & \\
\hline 6 & & & & & & & & 14 & & & 0 & 0 & 0 & 0 & $\equiv$ \\
\hline 7 & & 0 & & 0 & 0 & & & 15 & 0 & 0 & 0 & 0 & 0 & 0 & \\
\hline 8 & & 0 & 0 & 0 & 0 & 0 & & & $\because$ & $\therefore$ & $\therefore$ & $\therefore$ & $\therefore$ & $\because$ & 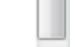 \\
\hline
\end{tabular}

Figure 17. The answer key of the produced exam-form. 


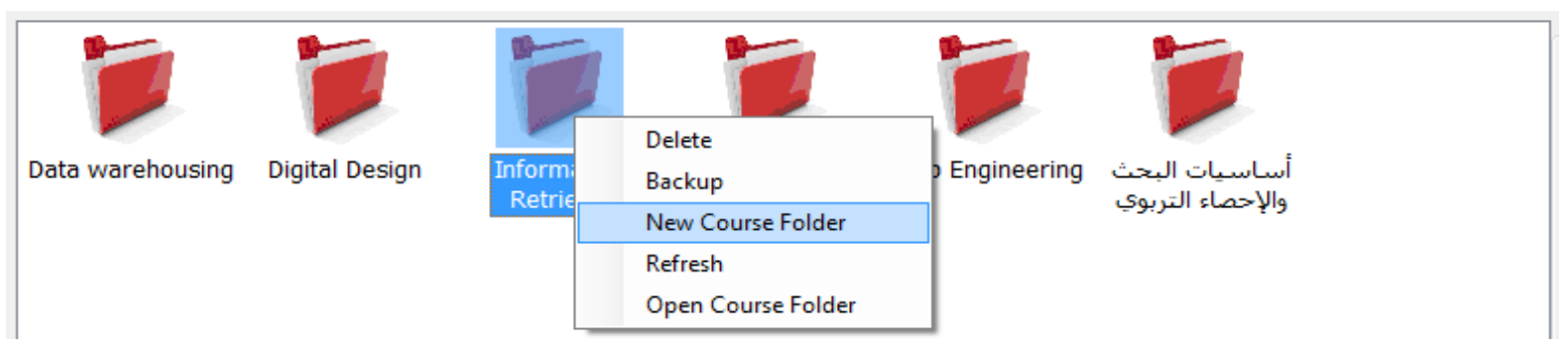

Figure 18. Manipulation of course questions banks.

4. In the following circuit, suppose that $A=00$ and $B=10, C 0=1$ then $S, C 2$ are:

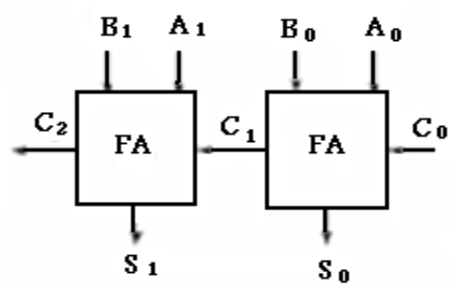

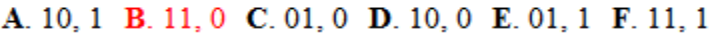

Figure 19. Example multiple-choice question with an inline figure.

figure as it appears in the appendix part of the form. e.g., it will be replaced by "Figure 5", or "figure x" in general. The sequence number " $\mathrm{x}$ " here is automatically generated by the software; the user does not have to worry about this issue.

\section{3) Unique Appendix Figures}

A figure can be inserted as a unique (not repeatable) figure at the end of the exam-form as appendix. That is; if the figure is referenced inside $n$ questions in the same exam-form, then this figure will appear only once in the appendix of the exam-form. Again, as in Repeatable appendix figures, the figure reference inside the question will be replaced by a proper reference to the figure as it appears in the appendix part of the exam-form. e.g., it will be replaced by "Figure 5", or "figure $\mathrm{x}$ " in general. The sequence number " $\mathrm{x}$ " here is automatically generated by the software. Again, the user does not have to worry about this issue.

\section{4) More Protection Features}

To protect already inserted questions (especially multiple-choice questions) from being mistakenly altered by the user, the user may want to add protection to that particular question from being edited.

$\mathrm{X}$-Pro Milestone is also equipped with

a) A simple, word-based spell checker.

b) A tool to insert symbols (e.g., $\Pi, \cap, \rightarrow, \ldots$ )

c) A tool to insert formatting tags that allows the user to enter entities such as equations and chemical formulas (such as $\mathrm{E}=\mathrm{MC}^{2}$ and $\mathrm{H}_{2} \mathrm{O}$ ).

\subsection{A Word about X-Pro Milestone User Friendliness}

Most important of all, X-Pro Milestone is user-friendly. According to [14], the most important features that identify user friendly software are the following. Those features were all considered when developing X-Pro Milestone.

- Simple to install, easy to remove, and easy to update: X-Pro Milestone is Windows-based; it can be installed into the system and can be safely removed through the Control Panel of the operating system. The user does not have to be expert to do these tasks. The software can be downloaded from the following link Http://sulieman.net/xpro/get. Updates are automatically detected and the user will be automatically notified of them.

- Intuitive, Pleasant, easy-to-navigate Graphical User Interface (GUI): all functions that the application provides are directly reachable through the application main menu. Controls and components are grouped 


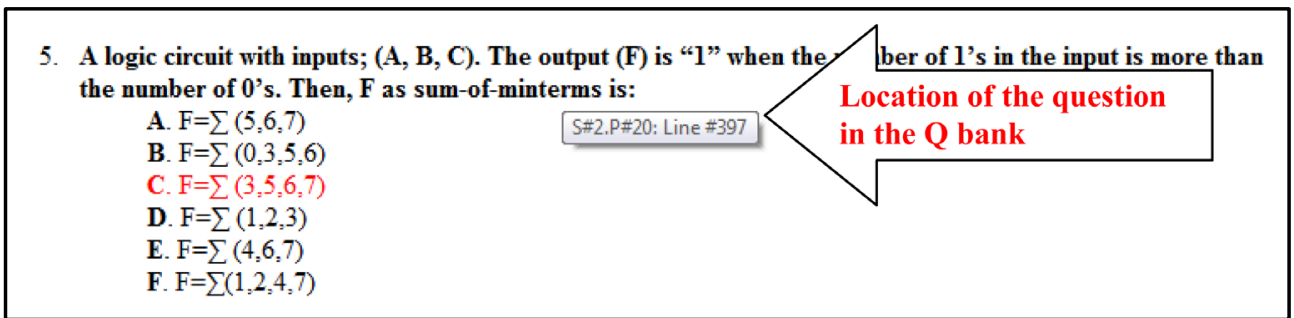

Figure 20. Example multiple-choice question with a popup indicating the location of the question in the question bank.

into tab pages on the same Windows form, which is the main screen of the application. This flat layout of the user interface helps the user have immediate and continuous interaction with those components and maximizes the user's benefit of all the functionalities provided by X-Pro Milestone.

- Doesn't need third-party software: X-Pro is stand-alone application. The user does not need database management system or any other difficult-to-find third-party software. The only third-party software the application requires is Microsoft's Internet Explorer, which automatically comes with the hosting environment; that is the Windows Operating system.

- Adheres to standards: The input of the application follows the Rich Text Format (RTF) standard. The output of the application; that is the exam-forms, is produced in the form of standard HTML and CSS formats. Both are well-known and widely-accepted standards.

- Effective error handling: If the user finds some typos or mistakes in any of the questions of the produced exam instance, then he/she can locate and directly navigate to the erroneous question. This is achieved by placing the mouse pointer on top of the question in the web browser or in the "Preview and Export Exam-Forms" tab page as shown in Figure 20. A balloon will pop up informing the user about the section number, the pool number and the line number where the question is placed within the course question bank.

As indicated in Figure 20 also, the correct answer of the multiple-choice question is displayed in red color (in preview-mode only, when the exam-form is printed out, all the choices will look and feel similar). This feature is needed if the user would like to double-check the correctness of the question.

\section{Conclusion}

In this paper we presented X-Pro milestone, a tool that enables instructors to construct a course-syllabus personalized question-bank of his/her own. X-Pro Milestone supports wide spectrum of question types (multiplechoice, true/false, short-answer essay questions, and free-response questions). The user can also produce exam instances with multiple forms based on a set of randomly selected set of pools. Those pools, in turn, are also randomly selected from within a set of pre-fed set of section. X-Pro Milestone has unique features that make it superior to other available software packages in the market. For instance, X-Pro Milestone question banks are personalized, portable, and highly customizable to fit user's needs. X-Pro Milstone is capable of automatically producing formatted and printable exam-forms along with an answer sheet and an answer key for each form. Putting the software into practice shows that it significantly reduces the effort required by the user to prepare and grade his/her exams. X-Pro Milestone is a cost-effective alternative to computer-based examination systems while maintaining the same level of quality assurance in terms of cheating prevention.

\section{Acknowledgements}

I would like to thank Prof. Ahmad Audeh from the faculty of Education at Yarmouk University, Jordan, for his long support and advices during the development of the software. I also would like to thank my colleague Dr. O. Dorgham from Al-Balqa Applied University for his kind support and observations on the software usability.

\section{References}

[1] Cen, G., Dong, Y.X., Gao, W.L., Yu, L.N., See, S., Wang, Q., Yang, Y. and Jiang, H.B. (2010) A Implementation of an Automatic Examination Paper Generation System. Mathematical and Computer Modeling, 51, 1339-1342.

http://dx.doi.org/10.1016/j.mcm.2009.11.010 
[2] Ministry of Higher Education—Jordan (2010) MOHE Statistics. The Ministry of Higher Education and Scientific Research. http://www.mohe.gov.jo

[3] Bani-Ahmad, S. and Audeh, A. (2010) Re-Engineering of Multiple-Choice Exam-Form Production Tools: CostEffective and Quality-Assurance Approach. Proceedings of the 2010 International Conference on Education and Information Technology (ICEIT), Chongqing, 17-19 September 2010, v3-260-v3-264. http://dx.doi.org/10.1109/iceit.2010.5608376

[4] Bani-Ahmad, S. (2010) The ExPro: A New Multiple-Choice-Based Exam-Form Production Package. The 8th Conference of the Faculty of Education, "Education in the Era of Alternatives", Yarmouk University, Irbid.

[5] Bani-Ahmad, S. and Audeh, A. (2010) Re-Engineering of Multiple-Choice Exam-Form Production Tools: CostEffective and Quality-Assurance Approach. Journal of Software Engineering, 4. [Journal Paper]

[6] Maharey, S. (2011) Higher Education: Challenges for Developing Countries. Commonwealth Education Partnerships. http://www.cedol.org/wp-content/uploads/2012/02/Steve-Maharey-article.pdf

[7] ProProfs Blog (2011) Essential Ways to Prevent Cheating in Online Assessments. http://www.proprofs.com/blog/2011/11/essential-ways-to-prevent-cheating-in-online-assessments/\#sthash.QxBAimru. dpufhttp://www.proprofs.com/blog/2011/11/essential-ways-to-prevent-cheating-in-online-assessments/

[8] Hutchinson, D. and Wells, J. (2013) An Inquiry into the Effectiveness of Student Generated MCQs as a Method of Assessment to Improve Teaching and Learning. Creative Education, 4, 117-125. http://dx.doi.org/10.4236/ce.2013.47A2014

[9] Fayyoumi, A., Mohammad, H. and Faris, H. (2013) Mobile Based Learning and Examination: Students and Instructors Perceptions from Different Arab Countries. Journal of Software Engineering and Applications, 6, 662-669. http://dx.doi.org/10.4236/jsea.2013.612079

[10] Fayyoumi, A. and Zarrad, A. (2014) Novel Solution Based on Face Recognition to Address Identity Theft and Cheating in Online Examination Systems. Advances in Internet of Things, 4, 5-12. http://dx.doi.org/10.4236/ait.2014.42002

[11] Johnston, S.P. and Huczynski, A. (2008) Textbook Publishers' Website Objective Question Banks: Does Their Use Improve Students' Examination Performance? Active Learning in Higher Education Journal, 7, 11-22.

[12] Burton, S., Sudweeks, R., Merril, P. and Wood, B. (1991) How to Prepare Better Multiple-Choice Test Items: Guidelines for University Faculty. Brigham Young University Testing Services.

[13] Pressman, R. and Maxim, B. (2014) Software Engineering: A Practitioner's Approach, 8th Edition. McGraw-Hill. Boston.

[14] Wallen, J. (2010) 10 Things That Make Software User-Friendly. TechRepublic. http://www.techrepublic.com/blog/10-things/10-things-that-make-software-user-friendly/

[15] Wikipedia. Iterative and Incremental Development. http://en.wikipedia.org/wiki/Iterative_and_incremental_development 